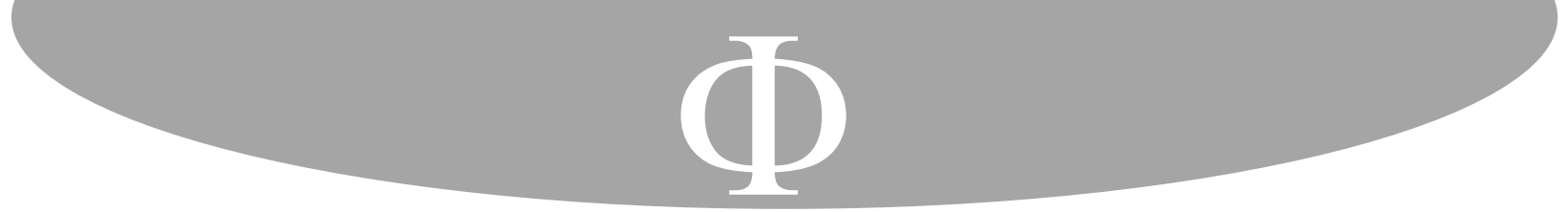

\title{
Edith Stein: de la concepción de la persona humana a la comprensión de la mujer $^{*}$
}

\section{(i) (2)

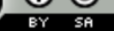

Para citar este artículo: Reyes-Gacitúa, Eva. «Edith Stein: de la concepción de la persona humana a la comprensión de la mujer». Franciscanum 175, Vol. 63 (2021): 1-23.

\section{Resumen}

Actualmente, «pensar la mujer» constituye una tarea inacabada y debatida. En pleno siglo XX, la fenomenóloga Edith Stein explora tal despliegue interrogándose por su naturaleza y valor propio. La presente investigación expone el itinerario que establece la filósofa desde la reflexión de la persona humana a la comprensión de la mujer.

Para tal efecto, se articulan desde la obra steiniana los principales conceptos y matices que, mediante el análisis fenomenológico posibilita examinar las nociones constituyentes del sujeto en su dimensión corporal y espiritual. Finalmente, se esboza la peculiaridad de la esencia femenina, cuya indagación permite detenerse en la pregunta esencial por la mujer. La conclusión avanza hacia un balance de los principales aspectos desarrollados como aporte al diálogo contemporáneo.

\section{Palabras clave}

Carne, mi cuerpo, alma, peculiaridad femenina, naturaleza de la mujer.

\section{Edith Stein: from the conception of the human person to the understanding of women}

\begin{abstract}
Currently, «wondering about women» is an unfinished and debated task. In the middle of the 20th century, the phenomenologist Edith Stein explores such a scenario by asking herself about its nature and value. The present research exposes the itinerary that the philosopher establishes from the reflection of the human person to the understanding of the woman.

\footnotetext{
* Artículo de reflexión. Patrocinado por la Vicerrectoría de Investigación, Dirección de Pastoral y Cultura Cristiana, Pontificia Universidad Católica de Chile. XVI concurso de investigación y creación para académicos. Investigador principal: Clemens Franken. Co-investigadores: María Teresa Stuven y Eva Reyes-Gacitúa.

** Doctora en Teología Dogmática por la Pontificia Universidad Católica de Chile. Académica Universidad Católica del Norte-Antofagasta, Chile. Investigadora del Centro de Estudios Interdisciplinarios en Edith Stein, UC. Contacto: evapamela@gmail.com. ORCID: https://orcid.org/0000-0002-5233-3785.
} 


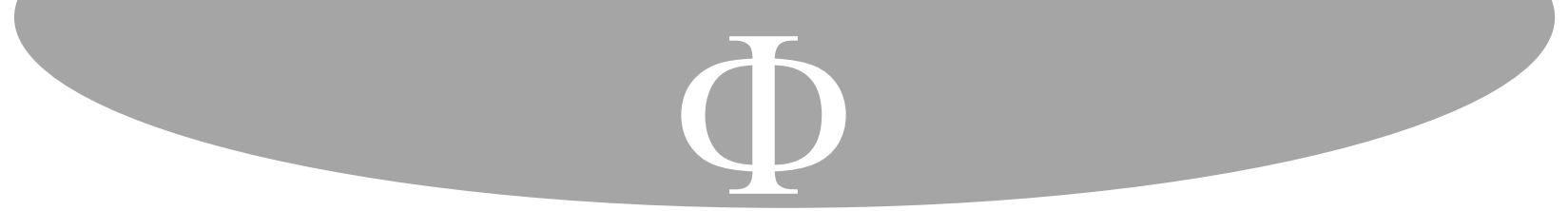

En otras palabras, establecer qué es la especie corresponde a la función epistemológica propia de la filosofía ${ }^{15}$. A la hora de definir la especie ser humano, Stein afirma:

Su carne es un cuerpo material, pero no sólo eso, sino a la vez un organismo que está formado y es operante desde el interior; y de nuevo el ser humano no es solamente organismo, sino un ser viviente animado, que de un modo particular perceptivamente- está abierto a sí mismo y al entorno; y finalmente es un ser espiritual, que se encuentra abierto cognoscitivamente a sí mismo y a los otros, y que puede libremente configurar a sí mismo y a los otros ${ }^{16}$.

Según la filósofa esta es la definición que muestra la estructura esencial del «núcleo de la persona», en cuanto todo a lo que se otorga el nombre de «persona humana» tiene en ella el centro de su $\operatorname{ser}^{17} \mathrm{y}$, en este sentido, sus diversos estratos se encuentran informados desde este núcleo ${ }^{18}$. Análogamente desde la tradición filosófica, según HannaBarbara Gerl-Falkovitz, «persona» significa algo doble: autoposesión y autodistancia. En palabras de Romano Guardini «persona» es el ser figurativo, interior, espiritual-creativo en la medida que descansa en sí mismo y dispone de sí. «Persona» significa que en mi ser yo mismo no puedo ser poseído por ninguna otra instancia, sino que me pertenezco a mí y soy una finalidad en sí19. En esta dirección Stein testifica que se encuentran estrechamente relacionadas: «vida emotiva» gemütsleben, el «núcleo de la persona» y la «profundidad del alma». Este núcleo remite a lo que es la persona en sí, o también la «esencia», «lo que mueve primeramente y en el fondo», a modo de primum movens ${ }^{20}$. Específicamente, «ese interior» del cual habla, «no se puede separar del todo que siente, piense y quiere, y que hace del cuerpo un cuerpo humano vivo y dotado de configuración personal» ${ }^{21}$. A partir de una bella expresión la filósofa enuncia:

Y si los curiosos, al borde del camino sólo distinguían una masa indiferenciada, sin embargo, para la madre o para la novia, aquel que ella espera es el ser único al que ningún otro es semejante: el misterio de su esencia del cual el amor de la madre o de la novia adivina algo, sólo la mirada de Dios que penetra todo, ante lo cual las medidas humanas de «grande»y de «pequeño», de «importante»y de «sin valor», pierden todo su significado 22 .

\footnotetext{
${ }^{15}$ Cf. Edith Stein, Problemas de la formación de la mujer. Obras Completas IV (Madrid: Ediciones El Carmen, Editorial de Espiritualidad, Editorial Monte Carmelo, 2003), 496.

${ }^{16}$ Edith Stein, Problemas de la formación de la mujer, 496.

${ }^{17} \mathrm{Cf}$. Edith Stein, Estructura de la persona humana, 669.

${ }^{18} \mathrm{Cf}$. Edith Stein, Estructura de la persona humana, 670.

${ }^{19}$ Cf. Hanna-Barbara Gerl-Falkovitz, Frau -Männin - Menschin. Zwischen Feminismus und Gender (Kevelaer: Butzon \& Bercker, 2009), 195.

${ }^{20} \mathrm{Cf}$. Beate Beckmann, Phänomenologie des religiösen Erlebnisses. Religionsphilosophische Überlegungen im Anschluss an Adolf Reinach und Edith Stein (Wurzburgo: Königshausen \& Neumann, 2003), 202.

${ }^{21}$ Edith Stein, Estructura de la persona humana, 707.

${ }^{22}$ Edith Stein, Ser finito y ser eterno. Obras Completas III (Madrid: Ediciones El Carmen, Editorial de Espiritualidad, Editorial Monte Carmelo, 2007), 1094.
} 


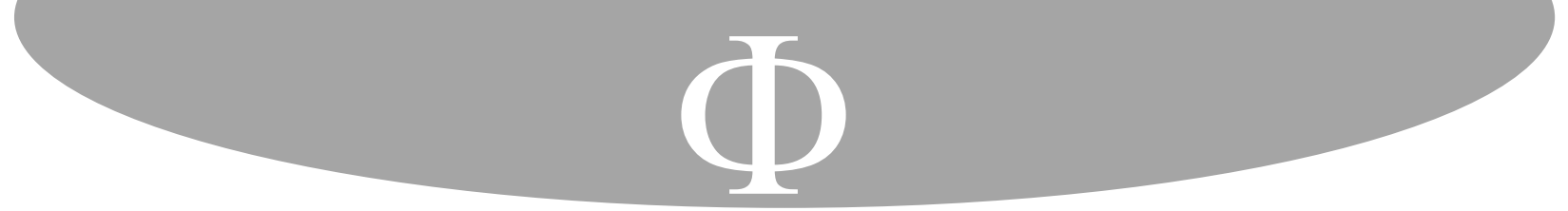

\section{Pensar la Mujer}

Según Edith Stein «pensar la mujer» es «una cuestión vivamente debatida», por tanto, se hace necesario entrar en su problemática desde el punto de vista de su situación actual $^{46}$. Al respecto observa: «existe una conexión extraña llena de contradicciones» entre la idea romántica de la mujer y aquella otra que valora sólo lo biológico entre actuales grupos de poder. Enfatiza:

Lo que aquí se da es una cancelación de la evolución de los últimos decenios y una limitación de la mujer a la actividad doméstica y familiar (...) No se tiene en cuenta aquí la esencia espiritual de la mujer, como tampoco las leyes de la evolución histórica (...) de esta manera considera: «se hace violencia al espíritu» ${ }^{47}$.

Entonces, tal escenario permite detenerse en la pregunta por la peculiaridad esencial de la mujer ${ }^{48}$, es decir, reflexionar si existe una «especie» universal de mujer, que comprenda una mayor diversidad de tipos e individuos y que, a su vez, difícilmente pueda hacer referencia a una situación común a ellas. Esto porque para el pensamiento steiniano es disímil la situación según la generación, el estado, el concepto del mundo, sin dejar de prescindir de la inabarcable pluralidad de los caracteres individuales, cuyas diferencias no pueden ser ignoradas en el tratamiento del tema ${ }^{49}$. En fin, no se ha estimado suficientemente la esencia de la mujer, ni se ha procurado dar una mirada sobre las acciones femeninas reales y concretas $^{50}$. Debido a esta inexactitud, Edith reconoce:

Hay hoy un gran número de hombres y de mujeres que se esfuerzan seriamente por conocer a fondo la peculiaridad y valor propio de la mujer, con los medios de la filosofía y teología, de la fisiología y psicología, de la sociología y de la historia de la cultura ${ }^{51}$.

A partir de esta consideración, profundiza desde dos vertientes, aportando significativamente al debate del género hoy. Por una parte, considera las diferencias de los sexos como algo históricamente condicionado por las relaciones exteriores, lo que podría ser superado por relaciones nuevas, y ver lo esencial en la naturaleza humana general, es decir, común a ambos sexos. La otra parte, tendría la convicción de una diferenciación según la esencia de la naturaleza humana ${ }^{52}$.

Lo elemental de esta formulación es que realiza una reflexión sobre la naturaleza de la mujer. Esta (naturaleza de la mujer), es el material humano dado en la experiencia misma, el cual no es algo unitario, sino diferenciado según los tipos e individuos ${ }^{53}$. Por

\footnotetext{
${ }^{46}$ Cf. Edith Stein, Problemas de la formación de la mujer, 458.

${ }^{47}$ Edith Stein, Problemas de la formación de la mujer, 467.

${ }^{48}$ Cf. Edith Stein, Problemas de la formación de la mujer, 463.

${ }^{49} \mathrm{Cf}$. Edith Stein, Problemas de la formación de la mujer, 458 y 459.

${ }^{50} \mathrm{Cf}$. Edith Stein, Problemas de la formación de la mujer, 467.

${ }^{51}$ Edith Stein, Problemas de la formación de la mujer, 468.

${ }^{52} \mathrm{Cf}$. Edith Stein, Problemas de la formación de la mujer, 468.

${ }^{53}$ Cf. Edith Stein, Problemas de la formación de la mujer, 473.
} 


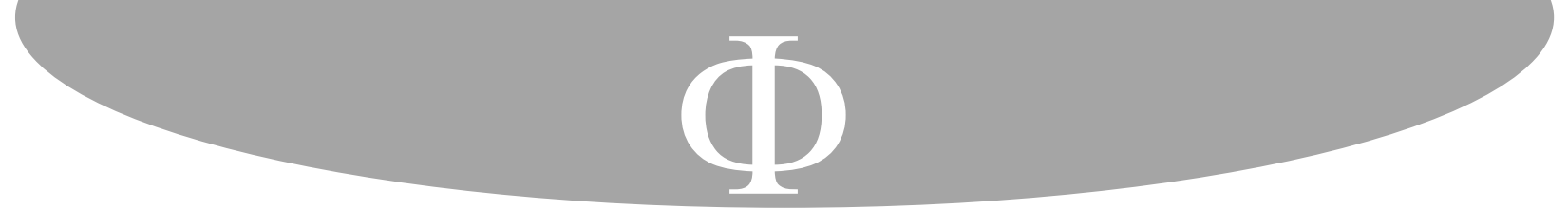

En efecto, para el pensamiento steiniano la naturaleza de la mujer, según el orden originario se encuentra al lado del hombre. En ella predomina en mayor medida la «respetuosa alegría ante las cosas creadas» ${ }^{103}$, refiriéndose a un conocimiento específico de los bienes, es decir, diferente de un mero conocimiento racional. Para Stein se trata de una fortaleza particular de la mujer, función espiritual propia en la cual radica claramente $^{104}$. Esta idea se conecta con la tarea que ella tiene en la protección y cuidado de la descendencia, en un sentido que la filósofa denomina «importancia de lo orgánico», abarcando al todo, incluso los valores específicos. Por lo tanto, es sensible y perspicaz con aquello que desea llegar a ser, crecer y desarrollarse. Es más, en esta relación hombremujer, Edith sitúa la cuestión del sacerdocio ministerial en la Iglesia, ella advierte la necesidad de edificar la realidad eclesial con una contribución activa, específicamente femenina ${ }^{105}$.

Fundamentalmente en esta línea de interpretación, para Hanna-Barbara GerlFalkovitz el lugar desde el cual emerge en primer lugar y más claramente el ser femenino es en cuanto a la disposición a concebir y la maternidad. Estas son las capacidades corporales de la especie mujer. Por ende, ambas cualidades portan afirmaciones sobre un 'interior' anímico, así la primera profesión de la mujer es la procreación y educación de la descendencia y al varón le es dado la protección. De esta forma, en la mujer se destacan las capacidades de conservar lo que está gestando-creciendo y de cuidar-fomentar su desarrollo ${ }^{106}$. Así, según Edith Stein, la unión de cuerpo y alma se da de una manera más íntima en la mujer:

Me parece que el alma de la mujer vive y está presente con mayor fuerza en todas las partes del cuerpo, y que en consecuencia queda afectada interiormente por todo aquello que le ocurre al cuerpo, mientras que en el hombre tiene más fuertemente el carácter de instrumento que le sirve en su actuación, lo cual conlleva un cierto distanciamiento consigo mismo. Todo esto se encuentra ciertamente en conexión con la misión de la mujer a la maternidad ${ }^{107}$.

Este concepto involucra una de las ideas más centrales del pensamiento steiniano, es decir, la alteridad ${ }^{108}$. A condición de que ella vive más desde el Gemüt, potencia que constituye su hábitat natural ${ }^{109}$. Por eso Stein habla del alma de la mujer y la distinción

\footnotetext{
${ }^{103}$ Edith Stein, Conferencias (1926-1933), 285.

${ }^{104}$ Cf. Edith Stein, Conferencias (1926-1933), 285.

${ }^{105}$ Cf. Irma Camarero, La doctrina del amor humano en santo Tomás de Aquino. Correlaciones con algunos aspectos de la doctrina sobre la empatía en Edith Stein (Roma: Ateneo Pontificio Regina Apostolorum, 2009), 152.

106 Cf. Hanna-Barbara Gerl-Falkovitz. Unerbittliches Licht. Versuche zur Philosophie und Mystik Edith Steins, 180 .

${ }^{107}$ Edith Stein, Conferencias (1926-1933), 320.

${ }^{108}$ Cf. Anneliese Meis, «La cuestión de la especificidad de la mujer en Edith Stein (1891-1942)», Teología y Vida 4, Vol. 50 (2009): 749.

${ }^{109}$ Cf. Alejandro Bertolini, Empatía y Trinidad en Edith Stein. Fenomenología, teología y ontología en clave relacional (Salamanca: Secretariado Trinitario, 2013), 201.
} 


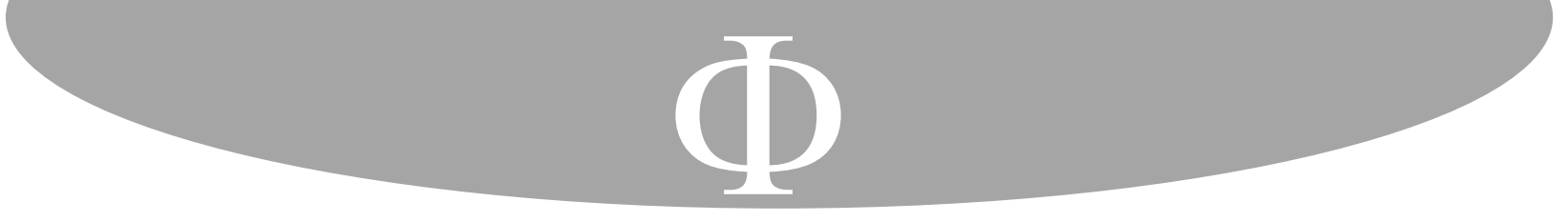

Para Stein la emotividad es centro del alma femenina. Por esta razón, la «formación de lo emotivo» deberá colocarse en el centro de la formación de las mujeres la que, sin embargo, requiere «claridad del entendimiento». Sólo quien es conmovido por la vida pondrá en movimiento su emotividad y quien desee despertarla, deberá ponerla en contacto con algo que le impacte ${ }^{117}$. En consecuencia, se puede encontrar diversas profesiones de mujeres, las cuales son ejercidas de forma sobresaliente, esto no quiere decir, que se trate de actividades específicamente femeninas, pues para la filósofa «no toda mujer es una personificación pura de la esencia femenina» ${ }^{118}$. «De hecho no existe, para Edith Stein, ninguna limitación fundamental de la mujer a la familia o a determinadas profesiones "femeninas", a pesar de que las ve tendencialmente en la mujer médico o en la profesora» ${ }^{119}$. Desde luego Stein se extiende hacia las tareas inmediatas de la legislación y de administración, ya que estas exigen la colaboración femenina para aconsejar, decidir y adoptar resoluciones. Sobre todo, en aquellas materias que afectan en primer lugar a mujeres. Pues la «mujer genuina», se encuentra cerca de las personas para no proceder abstracta y formalmente, sino para ponerse en las circunstancias vitales concretas y tenerlas en cuenta ${ }^{120}$. En esto despunta la vocación natural de la mujer, con múltiples vías abiertas para su realización ${ }^{121}$. Stein ha afirmado «ninguna mujer es sólo mujer», ya que se tienen grandes elementos en común con el hombre, por tal razón en la educación el talento natural indicará lo que cada chica debe elegir y entonces, podrá configurarlo a su manera $^{122}$.

A modo de sumario, la alteridad es el concepto que involucra una de las ideas más centrales del pensamiento steiniano. A condición de que la mujer vive más desde el Gemüt, lugar desde el cual emerge su ser femenino. Este se encuentra dado en la disposición a concebir y la maternidad por su mediación con lo corporal. Sin embargo, para la filósofa se trata de una disposición -que no es sólo biológica- y se transforma esencialmente en empatía. Para Stein no hay límites en la mujer.

\section{Conclusiones}

A partir de un interés antropológico filosófico, Edith Stein plantea desde la concepción de la persona humana, un tema frecuentemente desplazado, a saber, la comprensión de la naturaleza de la mujer. Ella asume críticamente este enfoque y piensa que generalmente desde factores políticos y sociales, se desprenden opiniones en las cuales se cree saber qué es una mujer. Este a priori, entrega una falsa generalización a la hora de «pensar la mujer» e incluso se manifiesta contrario al orden natural. Esta

\footnotetext{
117 Cf. Edith Stein, Conferencias (1926-1933), 327.

118 Edith Stein, Conferencias (1926-1933), 334-335.

${ }^{119}$ Hanna-Barbara Gerl-Falkovitz, «La cuestión de la mujer según Edith Stein», 780.

${ }^{120}$ Cf. Edith Stein, Conferencias (1926-1933), 339.

${ }^{121}$ Cf. Irma Camarero, La doctrina del amor humano en santo Tomás de Aquino. Correlaciones con algunos aspectos de la doctrina sobre la empatía en Edith Stein, 153.

122 Cf. Edith Stein, Apéndices, 1141.
} 
